\title{
Shikonin induces mitochondria-mediated apoptosis and attenuates epithelial-mesenchymal transition in cisplatin-resistant human ovarian cancer cells
}

\author{
KRISTINA SHILNIKOVA ${ }^{1}$, MEI JING PIAO ${ }^{1}$, KYOUNG AH KANG ${ }^{1}$, YEA SEONG RYU ${ }^{1}$, JEONG EON PARK ${ }^{1}$, \\ YU JAE HYUN ${ }^{1}$, AO XUAN ZHEN ${ }^{1}$, YONG JOO JEONG ${ }^{2}$, UHEE JUNG ${ }^{3}$, IN GYU KIM ${ }^{4}$ and JIN WON HYUN ${ }^{1}$ \\ ${ }^{1}$ Department of Biochemistry, School of Medicine, Jeju National University, Jeju 63243; ${ }^{2}$ Department of Bio \\ and Nanochemistry, Kookmin University, Seoul 02707; ${ }^{3}$ Radiation Biotechnology Research Division, Korea \\ Atomic Energy Research Institute, Jeongeup, Jeollabuk 56212; ${ }^{4}$ Department of Radiation Biology, Environmental \\ Radiation Research Group, Korea Atomic Energy Research Institute, Daejeon 34057, Republic of Korea
}

Received April 3, 2017; Accepted August 8, 2017

DOI: $10.3892 / \mathrm{ol} .2018 .8065$

\begin{abstract}
Cisplatin-based chemotherapy often results in the development of chemoresistance when used to treat ovarian cancer, which is difficult to overcome. The present study investigated the cytotoxic and anti-migratory effects of shikonin, a naphthoquinone compound, on cisplatin-resistant human ovarian cancer A2780 cells (A2780-CR). Shikonin had a potent dose-dependent cytotoxic effect on A2780-CR cells, with $9 \mu \mathrm{M}$ shikonin treatment reducing A2780-CR cell viability by $50 \%$, validate using an MTT assay. Shikonin induced apoptosis, as evidenced by the increased number of apoptotic bodies, following staining with Hoechst 33342, and terminal deoxynucleotidyl cell transferase dUTP nick end labeling-positive cells following treatment. Flow cytometry and fluorescent microscope imaging, following JC-1 staining, revealed that shikonin increased mitochondrial membrane depolarization. Also it altered the levels of apoptosis-associated proteins, leading to diminished expression of $\mathrm{B}$ cell lymphoma-2 (Bcl-2), enhanced expression of Bcl-associated $\mathrm{X}$, and cleavage of caspase- 9 and -3 , as revealed using western blot analysis. Shikonin activated mitogen-activated protein kinases; while treatment with specific inhibitors of these kinases attenuated the decline in cell viability induced by shikonin treatment. In addition, the cell migration assay and western blot analysis indicated that shikonin decreased the migratory capacity of A2780-CR cells via the upregulation of epithelial-cadherin and downregulation of neural-cadherin.
\end{abstract}

Correspondence to: Professor Jin Won Hyun, Department of Biochemistry, School of Medicine, Jeju National University, 102 Jejudaehakro, Jeju 63243, Republic of Korea

E-mail: jinwonh@jejunu.ac.kr

Key words: shikonin, drug resistance, epithelial-mesenchymal transition, human ovarian cancer, mitochondria-mediated apoptosis, mitogen-activated protein kinases
Taken together, the results of the present study indicated that shikonin induces mitochondria-mediated apoptosis and attenuates the epithelial-mesenchymal transition in A2780-CR cells.

\section{Introduction}

Ovarian cancer is the most aggressive gynecologic cancer and is thus the main cause of cancer-associated mortality in women worldwide $(1,2)$. According to a report by the American Cancer Society, the standard treatment approach is a platinum compound, for example cisplatin or carboplatin, in combination with a taxane, for example paclitaxel or docetaxel; however, intrinsic or acquired tumor chemoresistance remains a serious clinical problem and a major obstacle to successful therapy (3). Although rates of response of the primary tumor to taxane- and platinum-based therapy are high, $20 \%$ of cases remain non-responsive and the majority of patients will relapse and ultimately succumb to drug-resistant disease (4). The anticancer effects of platinum-based antineoplastics are mediated by their ability to generate irreparable intrastrand DNA crosslinks/adducts, which result in the induction of apoptosis and initiation of oxidative and endoplasmic reticulum stress (5). A number of factors lead to drug resistance, including enhanced drug efflux, drug inactivation, modifications of drug targets, processing of drug-induced damage and avoidance of apoptosis $(6,7)$. Furthermore, platinum resistance in cancer arises from numerous adaptive mechanisms, including decreased cellular uptake, enhanced DNA repair and tolerance and inactivation by glutathione; however, the overall molecular mechanisms require further elucidation (4).

Shikonin is a natural compound obtained from the roots of Lithospermum erythrorhizon, Arnebia euchroma and Onosma paniculata (8-10). Shikonin has anti-inflammatory, anti-oxidant, anticancer, wound-healing and anti-microbial effects (11). Shikonin induces cancer cell death via various mechanisms, including suppression of the activity of protein tyrosine kinases and DNA topoisomerases, which have an important function in gene regulation and the inhibition 
of expression of tumor necrosis factor receptor-associated protein 1. The anticancer effect of shikonin is associated with enhanced expression of tumor protein p53 and suppression of cancer cell glycolysis through targeting of pyruvate kinase M2 (11,12). Previous studies have demonstrated that shikonin induces apoptosis in keratinocyte HaCaT cells, human stomach carcinoma AGS cells, colon cancer cells, lung cancer cells, human medullary thyroid carcinoma cells, human promyelocytic leukemia HL-60 cells, and human cervical cancer HeLa cells (13-20). The present study investigated the apoptotic effects of shikonin on cisplatin-resistant human ovarian cancer A2780 (A2780-CR) cells.

The epithelial-mesenchymal transition (EMT), a feature of aggressive tumors, is characterized by decreased epithelial (E-)cadherin expression and increased neural (N-)cadherin expression, which contributes to a stroma-oriented cellular adhesion profile with enhanced cancer cell motility and invasive features. These events have already been demonstrated in several cancer cell lines including breast, prostate and colorectal adenocarcinoma (21). Shikonin was reported to stimulate EMT in skin wound-healing (22). On the other hand, shikonin inhibits the migration and invasion of breast cancer and glioblastoma cells $(23,24)$. Therefore, the present study sought to clarify the effects of shikonin on the migratory capacity of ovarian cancer cells. On the basis of its cytotoxic and anti-migratory effects, shikonin may be an alternative drug to be used during chemotherapy to treat this type of tumor.

\section{Materials and methods}

Reagents. Shikonin [5,8-dihydroxy-2-(1-hydroxy-4-methylpent-3-enyl)naphthalene-1,4-dione] was obtained from Cayman Chemical Company (Ann Arbor, MI, USA). MTT, Hoechst 33342, trypan blue solution, Triton X-100 solution and the anti-actin antibody (catalog no. A2066) were obtained from Sigma-Aldrich; Merck KGaA (Darmstadt, Germany). 5,5', 6,6'-Tetrachloro-1,1',3,3'-tetraethylbenzimidazolocarboc yanine iodide (JC-1) was obtained from Invitrogen (Thermo Fisher Scientific, Inc., Waltham, MA, USA). The anti-phosphorylated c-Jun N-terminal kinase (phospho-JNK) (catalog no. 9255), anti-JNK (catalog no. 9252), anti-phospho-p38 (catalog no. 9211), and anti-caspase-3 (catalog no. 9662) antibodies, SB203580 (p38 inhibitor), and U0126 (extracellular signal-regulated kinase (ERK) inhibitor) were provided by Cell Signaling Technology, Inc. (Danvers, MA, USA). The anti-B-cell lymphoma-2 (Bcl-2)-associated $\mathrm{X}$ protein (Bax) (catalog no. sc-7480), anti-Bcl-2 (catalog no. sc-492), anti-caspase-9 (catalog no. sc-7885), anti-phospho-extracellular-related kinase (ERK) (catalog no. sc-7383), anti-ERK (catalog no. sc-154), anti-p38 (catalog no. sc-535), anti-E-cadherin (catalog no. sc-7870), and anti-N-cadherin (catalog no. sc-7939) antibodies were obtained from Santa Cruz Biotechnology, Inc. (Dallas, TX, USA). The JNK inhibitor SP600125 was purchased from Selleck Chemicals (Houston, TX, USA). All other chemicals and reagents used were of analytical grade.

Cell culture. Cisplatin-sensitive and -resistant human ovarian carcinoma A2780 cells were purchased from Sigma-Aldrich; Merck KGaA). Paclitaxel-sensitive and -resistant (PR) human ovarian carcinoma SKOV3 cells were obtained from the MD Anderson Cancer Center (Houston, TX, USA). Cells were incubated at $37^{\circ} \mathrm{C}$ in a humidified atmosphere of $5 \% \mathrm{CO}_{2}$ and cultured in Dulbecco's modified Eagle's medium containing $10 \%$ heat-inactivated fetal bovine serum (both, Gibco; Thermo Fisher Scientific, Inc.), streptomycin (100 mg/ml), and penicillin $(100 \mathrm{U} / \mathrm{ml})$.

Cell viability. To determine the effect of shikonin on cell viability, A2780, A2780-CR, SKOV3 and SKOV3-PR cells were seeded in 24-well plates at a density of $1 \times 10^{5}$ cells $/ \mathrm{ml}$ and treated with $0.0,2.5,5.0,10.0,20.0,30.0$ or $40.0 \mu \mathrm{M}$ shikonin for $48 \mathrm{~h}$. Cells were treated with shikonin under room-temperature conditions and subsequently incubated at $37^{\circ} \mathrm{C}$. To investigate the protective effect of mitogen-activated protein kinase (MAPK) inhibitors against shikonin-induced cell death, A2780-CR cells were pretreated with $10.0 \mu \mathrm{M}$ SB203580, U0126, or SP600125 at room temperature, incubated for $1 \mathrm{~h}$ at $37^{\circ} \mathrm{C}$, and subsequently treated with $9.0 \mu \mathrm{M}$ shikonin and incubated for another $24 \mathrm{~h}$. MTT stock solution (125 $\mu \mathrm{l} ; 2 \mathrm{mg} / \mathrm{ml})$ was then added into each well to attain a total reaction volume of $500 \mu \mathrm{l}$. Following incubation for $4 \mathrm{~h}$ at $37^{\circ} \mathrm{C}$, plates were centrifuged at $200 \mathrm{x}$ g for $10 \mathrm{~min}$ at room temperature and the supernatants were aspirated. The formazan crystals in each well were dissolved in $300 \mu$ l dimethyl sulfoxide, and absorbance at $540 \mathrm{~nm}$ was read on a scanning multi-well spectrophotometer (25). From the cell viability at various concentrations of shikonin, the half maximal inhibitory concentration $\left(\mathrm{IC}_{50}\right)$ value was determined.

Colony formation. To examine the inhibitory effect of shikonin on colony-forming ability, A2780-CR cells were seeded in 60-mm dishes at a density of 400 cells/dish and treated with $9 \mu \mathrm{M}$ shikonin on day 7 of incubation. The medium was changed every 3 days. After 11 days, cells were fixed using $70 \%$ ethanol for $10 \mathrm{~min}$ at room temperature and stained with trypan blue solution $(0.4 \%)$ at room temperature until blue colonies became visible. Colony-forming units (CFUs) were considered formed if they were visible to the naked eye. CFUs were imaged and counted using Image $\mathbf{J}$ software (version 1.47; National Institutes of Health, Bethesda, MD, USA).

Terminal deoxynucleotidyl transferase-mediated dUTP nick end labeling (TUNEL) assay. To visualize apoptotic bodies, A2780-CR cells were seeded in chamber slides at a density of $1 \times 10^{5}$ cells $/ \mathrm{ml}$, incubated for $24 \mathrm{~h}$, and then treated with $9 \mu \mathrm{M}$ shikonin for $24 \mathrm{~h}$. Slides were then prepared according to the protocol of the DeadEnd Colorimetric TUNEL system (Promega Corporation, Madison, WI, USA). Cells were fixed in $10 \%$ buffered formalin for $25 \mathrm{~min}$ at room temperature, washed twice with PBS for $5 \mathrm{~min}$ at room temperature and permeabilized by immersing the slides in $0.2 \%$ Triton X-100 solution in PBS for $5 \mathrm{~min}$ at room temperature. Subsequently, cells were washed twice with PBS for 5 min at room temperature, covered with $100 \mu$ l equilibration buffer provided in a DeadEnd colorimetric TUNEL system (Promega Corporation) and equilibrated at room temperature for $10 \mathrm{~min}$. The rTdT reaction mix (containing 98\% equilibration buffer, $1 \%$ biotinylated nucleotide mix and $1 \%$ rTdT enzyme, all provided in a DeadEnd colorimetric TUNEL system) was 
added to the sections on a slide and the slide was covered with a plastic coverslip and incubated at $37^{\circ} \mathrm{C}$ for $60 \mathrm{~min}$ inside a humidified chamber. The plastic coverslips were removed and the reactions were terminated by immersing the slides in $2 \mathrm{X}$ saline-sodium citrate (SSC; made by dilution of 20X SSC available in a DeadEnd colorimetric TUNEL system with deionized water) for $15 \mathrm{~min}$ at room temperature. Following washing the slides three times in PBS for $5 \mathrm{~min}$ at room temperature, the slides were immersed in $0.3 \%$ $\mathrm{H}_{2} \mathrm{O}_{2}$ in PBS for between 3 and $5 \mathrm{~min}$ at room temperature, and washed three times in PBS. Subsequently, horseradish peroxidase-labeled streptavidin in a DeadEnd colorimetric TUNEL system (dilution, 1:500 in PBS) was added to each slide and the slides were incubated for $30 \mathrm{~min}$ at room temperature. Slides were washed three times in PBS and the chromogen solution (50 $\mu \mathrm{l}$ DAB Substrate 20X buffer, $950 \mu \mathrm{l}$ deionized water, $50 \mu \mathrm{l}$ DAB $20 \mathrm{X}$ chromogen and $50 \mu \mathrm{l} \mathrm{H}_{2} \mathrm{O}_{2}$ 20X, all except the deionized water provided in a DeadEnd colorimetric TUNEL system) were added to each slide and developed until there was a light brown background. Cells were washed four times in deionized water and the stained cells were mounted onto microscope slides in faramount aqueous mounting medium (Dako; Agilent Technologies, Inc., Santa Clara, CA, USA). Microscopic images of 5 fields of view per group (magnification, x20) were captured using the laser scanning microscope 5 PASCAL program (Carl Zeiss, AG, Oberkochen, Germany) on a confocal fluorescent microscope.

Nuclear staining with Hoechst 33342. A2780-CR cells were treated with $9 \mu \mathrm{M}$ shikonin and incubated for $24 \mathrm{~h}$. Next, $1.5 \mu \mathrm{l}$ Hoechst 33342 (stock solution, $10 \mathrm{mg} / \mathrm{ml}$ ), a DNA-specific fluorescent dye, was added to each well and incubated for $10 \mathrm{~min}$ at $37^{\circ} \mathrm{C}$. The stained cells were then observed under a fluorescence microscope, which was equipped with a Cool SNAP-Pro color digital camera and microscopic images were collected using a laser scanning microscope 5 PASCAL program. The index of apoptotic bodies was identified by dividing the number of observed apoptotic bodies (calculated manually for each microscopic image) by the number of unmodified nuclei observed on the same image. This method was also used to visualize the protective effect of MAPK inhibitors against shikonin-induced apoptosis. In this case, cells were pretreated with $10 \mu \mathrm{M}$ SB203580, U0126, or SP600125 and then treated with $9 \mu \mathrm{M}$ shikonin $1 \mathrm{~h}$ following this, as aforementioned. They were then stained with Hoechst 33342, as described.

Mitochondrial membrane potential $\left(\Delta \psi_{m}\right)$. For image analysis, following incubation and treatment with $9 \mu \mathrm{M}$ shikonin, A 2780-CR cells were stained with JC-1 $(10 \mu \mathrm{g} / \mathrm{ml})$ for $15 \mathrm{~min}$ at $37^{\circ} \mathrm{C}$ and affixed to microscope slides in mounting medium. The intensity of fluorescence emission in the red and green range was assessed using a confocal microscope and the Laser Scanning Microscope 5 PASCAL program. This experiment was validated using FAC-SCalibur flow cytometer (BD Biosciences, Franklin Lakes, NJ, USA). Shikonin (9 $\mu \mathrm{M})$-treated or untreated A2780-CR cells were harvested, washed, suspended in PBS containing JC-1 $(10 \mu \mathrm{g} / \mathrm{ml})$, incubated for $15 \mathrm{~min}$ at $37^{\circ} \mathrm{C}$ and analyzed using flow cytometry. The intensity of emission in the green range was assessed based on histograms generated by CellQuest Pro 5.1 software (Becton-Dickinson, Bedford, MA, USA) and ModFit LT 3.2 software (Verity Software House, Topsham, ME, USA).

Western blotting. A2780-CR cells were harvested, washed with PBS, lysed on ice for 30 min using PRO-PREP ${ }^{\mathrm{TM}}$ protein extraction solution (Intron Biotechnology, Inc., Seongnam, South Korea), and then centrifuged at $13,000 \mathrm{x}$ g for $15 \mathrm{~min}$ at $4^{\circ} \mathrm{C}$. The supernatants were collected, and the protein concentrations were measured using the Bio-Rad protein assay reagent kit (Bio-Rad Laboratories, Inc., Hercules, CA, USA). and equalized across samples by addition of suitable volumes of 5 X SDS loading dye and PBS. Aliquots of the lysates ( $40 \mu \mathrm{g}$ of protein) were boiled for $5 \mathrm{~min}$ and electrophoresed in $10 \%$, 12 , or $15 \%$ SDS-PAGE. The proteins in the gels were transferred onto nitrocellulose membranes (Bio-Rad Laboratories, Inc.), which were blocked with $2 \%$ fetal bovine serum for $1 \mathrm{~h}$ at room temperature and then incubated with the appropriate primary antibodies (dilution, 1:1,000) for $1 \mathrm{~h}$ at room temperature and subsequently overnight at $4^{\circ} \mathrm{C}$ to achieve optimal binding. Actin or COX4 were used as the loading control for total or mitochondrial protein fractions, respectively. The membranes were further incubated with secondary horseradish peroxidase-conjugated goat anti-rabbit immunoglobulin G (IgG; catalog no. G-21234) or goat anti-mouse IgG (catalog no. G-21040) antibodies (Pierce; Thermo Fisher Scientific, Inc.; dilution, 1:5,000) for $1 \mathrm{~h}$ at room temperature. Protein bands were visualized using an enhanced chemiluminescence western blotting detection kit (GE Healthcare Life Sciences, Little Chalfont, UK) and then exposed to X-ray film.

Cell migration assay. A2780-CR cells were seeded in $100-\mathrm{mm}$ dishes at a density of $1 \times 10^{4}$ cells $/ \mathrm{ml}$ and incubated for $24 \mathrm{~h}$. Next, following treatment with $9 \mu \mathrm{M}$ shikonin, a line (diameter, $1.5 \mathrm{~mm}$ ) was scratched across the bottom of each dish. After $48 \mathrm{~h}$ incubation, this area was observed under a fluorescence microscope.

Statistical analysis. All measurements were performed in triplicate, and all values represent the mean \pm standard error of the mean. The results were assessed using a one-way analysis of variance with Tukey's post-hoc test to analyze the differences. $\mathrm{P}<0.05$ was considered to indicate a statistically significant difference.

\section{Results}

Cytotoxic effect of shikonin on ovarian cancer cells. Shikonin exerted a potent, dose-dependent cytotoxic effect on cisplatin-sensitive and -resistant human ovarian carcinoma A2780 cells (Fig. 1A). The $\mathrm{IC}_{50}$ of shikonin was $11 \mu \mathrm{M}$ for $\mathrm{A} 2780$ cells and $9 \mu \mathrm{M}$ for A2780-CR cells. Thus, cisplatin-sensitive and cisplatin-resistant cells were highly sensitive to this compound. In addition, the effect of shikonin on the viability of paclitaxel-sensitive and -resistant human ovarian carcinoma SKOV3 cells was assessed (Fig. 1A). SKOV3-PR cells demonstrated lower sensitivity to shikonin than SKOV3 cells $\left(\mathrm{IC}_{50}\right.$ value 16 and $11 \mu \mathrm{M}$, respectively). Since cisplatin-resistant cells exhibited the greatest sensitivity to shikonin, A2780-CR cells were used in future experiments. To confirm the cytotoxic 


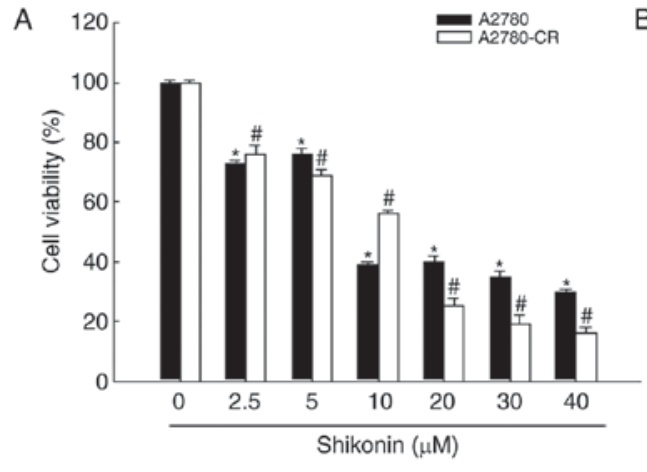

B
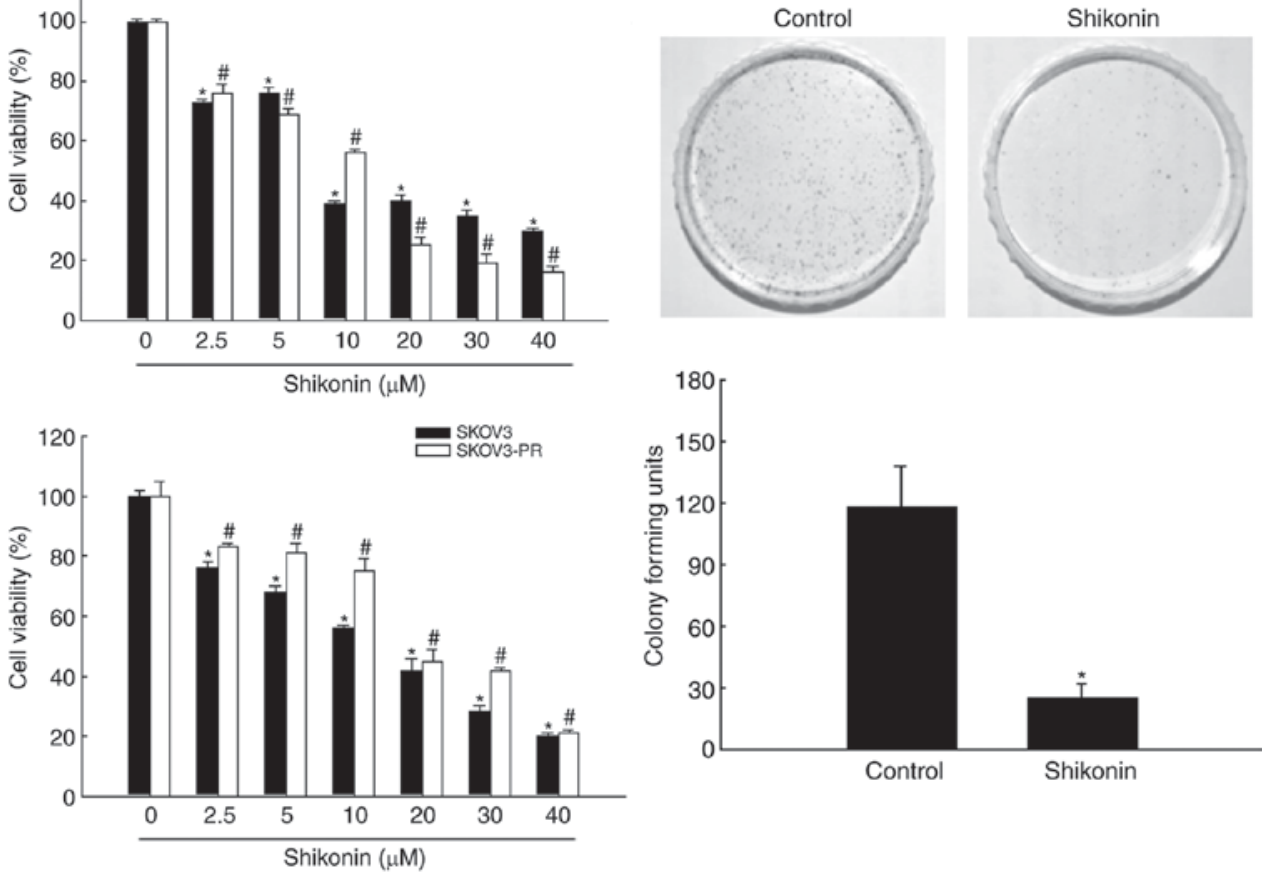

Figure 1. Shikonin induces death of ovarian cancer cells. (A) A2780, A2780-CR, SKOV3 and SKOV3-PR cells were treated with 0.0, 2.5, 5.0, 10.0, 20.0, 30.0 or $40.0 \mu \mathrm{M}$ shikonin for $48 \mathrm{~h}$. Cell viability was measured using the MTT assay. (B) For the colony formation assay, A2780-CR cells were cultured for 11 days and treated with $9 \mu \mathrm{M}$ shikonin on the seventh day. Colonies containing $>400$ cells were counted. ${ }^{*} \mathrm{P}<0.05$ and ${ }^{*} \mathrm{P}<0.05$, compared with the respective control groups. CR, cisplatin-resistant; PR, paclitaxel-resistant.

A
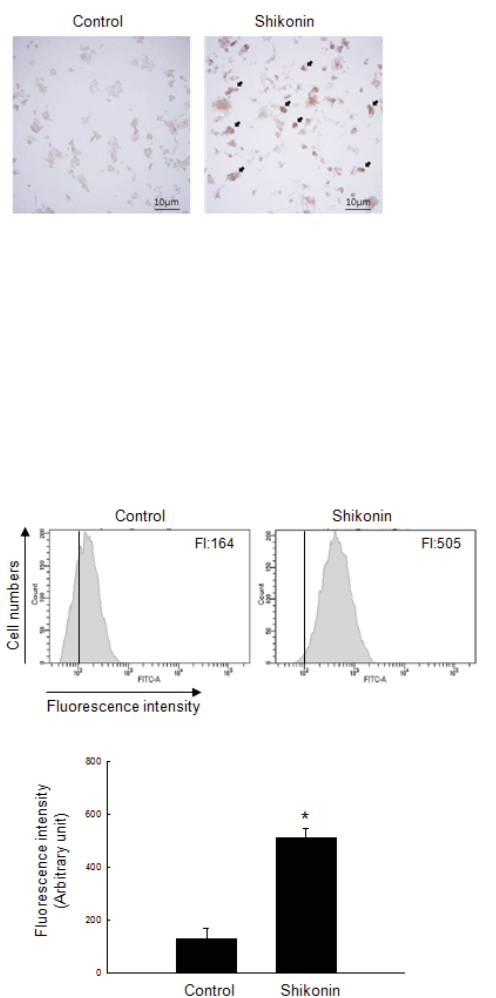

B
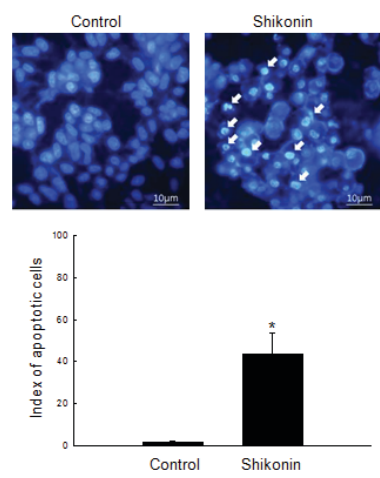

E

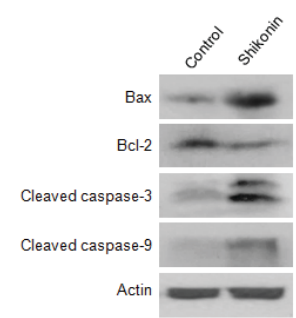

$c$

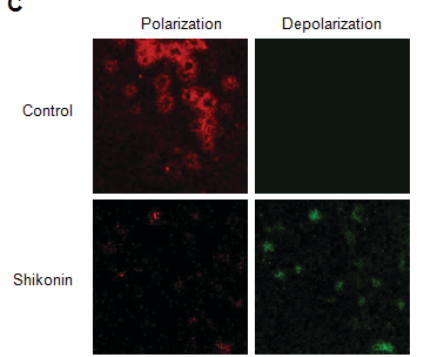

$\mathbf{F}$

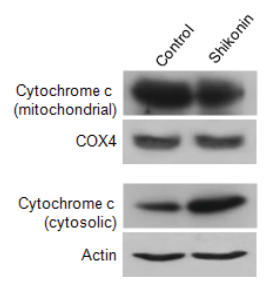

Figure 2. Shikonin induces apoptosis via the mitochondrial-dependent pathway in A2780-CR ovarian cancer cells. (A) Apoptosis in A2780-CR cells was examined by the TUNEL assay. Representative images are shown and TUNEL-positive cell nuclei are indicated by arrows. (B) Apoptotic bodies (arrows) were observed by fluorescence microscopy in A2780-CR cells stained with Hoechst 33342 and quantified. ${ }^{*} \mathrm{P}<0.05$. Cells were stained with JC-1 and $\Delta \psi \mathrm{m}$ and assessed by (C) confocal microscopy and (D) flow cytometry. (E) Cell lysates were subjected to western blot analysis for Bax, Bcl-2, cleaved caspase-3, and cleaved caspase-9. Actin was used as a loading control. (F) Western blot analysis was performed using an antibody against cytochrome c. COX4 and actin were used as loading controls for the mitochondrial and cytosolic fractions, respectively. CR, cisplatin-resistant; $\Delta \psi \mathrm{m}$, mitochondrial membrane potential; Bcl-2, B-cell lymphoma-2; Bax, Bcl-associated X; COX4, cyclooxygenase 4. 


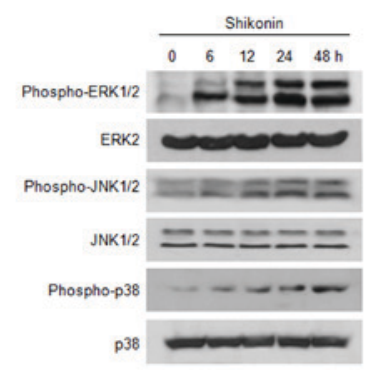

C

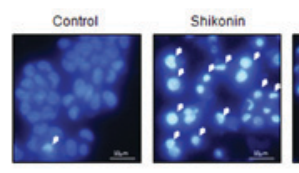

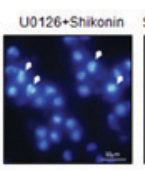

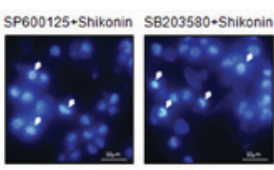

B

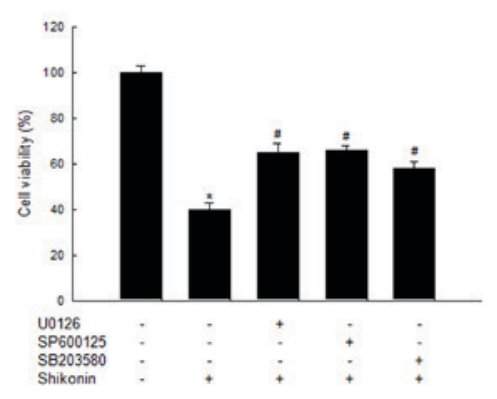

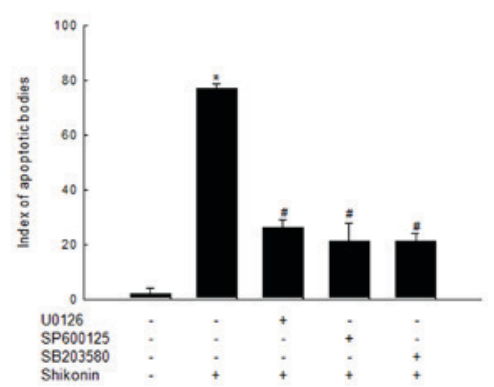

Figure 3. Effect of shikonin on the MAPK signaling pathway. (A) Cells were harvested following treatment with $9 \mu \mathrm{M}$ shikonin for different durations $(0-48 \mathrm{~h})$ and subjected to western blot analysis using primary antibodies against phospho-ERK1/2, ERK2, phospho-JNK1/2, JNK1/2, phospho-p38, and p38. Following treatment with MAPK inhibitors and/or shikonin, (B) cell viability was assessed using the MTT assay and (C) apoptotic body formation was observed using Hoechst 33342 nuclear staining. Apoptotic bodies are indicated by arrows. " $\mathrm{P}<0.05$ vs. control cells; ${ }^{~} \mathrm{P}<0.05$ vs. cells treated with shikonin alone. MAPK, mitogen-activated protein kinase; ERK1/2, extracellular-related kinase 1/2.

effect of shikonin on A2780-CR cells, the colony-forming ability was determined (Fig. 1B). Treatment with $9 \mu \mathrm{M}$ shikonin significantly reduced the colony-forming ability of A2780-CR compared with control cells. Thus, $9 \mu \mathrm{M}$ shikonin was used for subsequent studies of A2780-CR cells.

Induction of apoptosis by shikonin in cisplatin-resistant ovarian cancer cells. To verify whether the cytotoxic properties of shikonin were caused by apoptosis, the degree of apoptotic body formation and the extent of DNA condensation were visualized using the TUNEL assay and Hoechst 33342 nuclear staining, respectively. Shikonin-treated cells exhibited a greater number of apoptotic bodies than control cells (Fig. 2A). Furthermore, the degree of DNA condensation and nuclear fragmentation were also significantly higher in the shikonin-treated group (Fig. 2B).

Involvement of the mitochondrial pathway in shikonin-induced apoptosis. As indicated by confocal microscopy, the mitochondria in control cells demonstrated bright red JC-1 fluorescence, signifying $\Delta \psi_{\mathrm{m}}$ polarization. By contrast, shikonin-treated cells had an attenuated red mitochondrial fluorescence and an enhanced green fluorescence, indicating $\Delta \psi_{\mathrm{m}}$ depolarization (Fig. 2C). Similar data were obtained by flow cytometry (Fig. 2D), confirming that shikonin decreased $\Delta \psi_{\mathrm{m}}$. The expression of proteins associated with mitochondria-induced apoptosis were also investigated. Treatment with shikonin decreased the level of the pro-survival/anti-apoptotic protein Bcl-2 and increased the level of pro-apoptotic protein Bax. Shikonin increased the levels of active (cleaved) caspase-9 and -3 (Fig. 2E). Thus, these data indicate that shikonin induces apoptosis via the mitochondrial pathway.
The mitochondrial apoptosis pathway is associated with releasing of cytochrome $c$ from mitochondria into the cytosol (26); therefore, the levels of cytochrome $c$ were detected using western blot analysis in the mitochondrial and cytosolic protein fractions separately (Fig. 2F). These results confirmed that this release indeed occurred following shikonin treatment.

Induction of apoptosis by shikonin via MAPK activation. Shikonin induced the expression of mitochondria-associated pro-apoptotic proteins; therefore, our group sought to investigate whether shikonin treatment activated the MAPK signaling cascade. Previous studies have demonstrated that shikonin activates the signaling network of ERK and protein kinase B $(11,15)$. In the present study, western blot analysis demonstrated that shikonin induced phosphorylation, and thus activation of, JNK, p38 and ERK in a time-dependent manner (Fig. 3A), indicating the involvement of this pathway in shikonin-induced cell death. Next, whether the specific inhibitors of JNK, p38, and ERK attenuated shikonin-induced apoptosis was assessed. The MTT assay and Hoechst 33342 nuclear staining indicated that the JNK inhibitor SP600125, the p38 inhibitor SB203580, and the ERK inhibitor U0126 reduced the cytotoxic effects of shikonin (Fig. 3B and C).

Effect of shikonin on the EMT in cisplatin-resistant ovarian cancer cells. To investigate the effect of shikonin treatment on the migratory capability of A2780-CR cells, lines (diameter, $1.5 \mathrm{~mm}$ ) were scratched across the bottom of culture dishes following treatment with $9 \mu \mathrm{M}$ shikonin, and the degree of cell migration was observed after $48 \mathrm{~h}$ incubation (Fig. 4A). Whereas the gap width diminished with time in untreated cells, it increased in shikonin-treated cells. The difference 


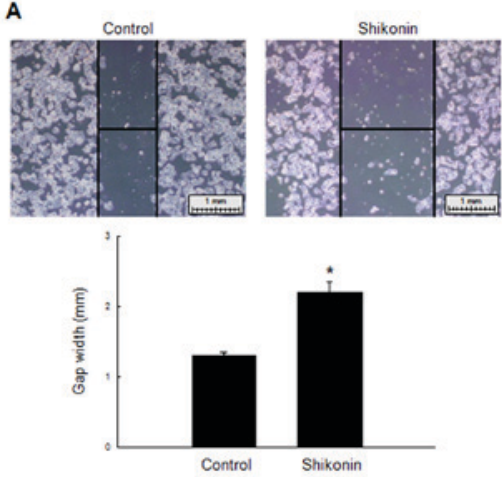

B

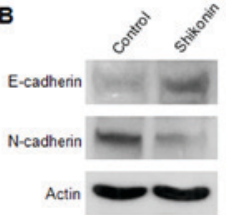

Figure 4. Effect of shikonin on the epithelial-mesenchymal transition in cisplatin-resistant ovarian cancer cells. (A) Cells were seeded in $100-\mathrm{mm}$ culture dishes and, following treatment with $9 \mu \mathrm{M}$ shikonin, a scratch (diameter, $1.5 \mathrm{~mm}$ ) was made along the bottom of the dish. The wound was observed after $48 \mathrm{~h}$ incubation. The gap distance was measured and all data were quantified. Representative images for $48 \mathrm{~h}$ incubation are shown. ${ }^{*} \mathrm{P}<0.05$ vs. control cells. (B) Cell lysates were electrophoresed and underwent western blot analysis with anti-E-cadherin and anti-N-cadherin antibodies. Actin was used as a loading control. E-cadherin, epithelial cadherin; N-cadherin, neural cadherin.

between the treated and control groups was significant at $48 \mathrm{~h}$. Thus, shikonin treatment attenuated the migration capability of A2780-CR cells. To further confirm this result, the expression of $\mathrm{E}$ - and $\mathrm{N}$-cadherin, which are glycoproteins that are required to initiate and stabilize cell-cell contact, was determined. The expression of E-cadherin and N-cadherin protein was increased and decreased, respectively, in the shikonin-treated group compared with the control group (Fig. 4B).

\section{Discussion}

A large proportion of therapeutic failures in the ovarian cancer treatment are associated with chemoresistance to cisplatin- or taxol-based therapy $(3,4)$. Overcoming cisplatin resistance remains difficult (4). The present data study demonstrated that shikonin reportedly inhibits the proliferation of cisplatin-resistant human ovarian cancer cells in a dose-dependent manner. Additionally, we attempted to clarify the molecular mechanisms by which shikonin induces apoptosis in A2780-CR cells.

In the present study, shikonin exhibited a greater cytotoxic effect in cisplatin-resistant cells compared with parental ovarian cancer A2780 cells, as evidenced by the respective $\mathrm{IC}_{50}$ values. Considering the antioxidant effect of shikonin (11), the difference in sensitivity to shikonin between A2780 and A2780-CR cells may be due to its reactive oxygen species (ROS)-scavenging properties. Parental and cisplatin-resistant ovarian cancer cells have different redox statuses, with basic level of ROS in A2780-CR cells was higher than that in parental A2780 cells, with shikonin reducing the enhanced
ROS levels in A2780-CR cells (data not shown). This disorder in redox balance of A2780-CR cells may provide further cellular stress and lead to cytotoxicity. This assumption is consistent with the results of other studies, which demonstrate an association between chemotherapy resistance and enhanced ROS levels in different cancer cells (27-29). Apoptosis is a type of physiological cell death that is associated with specific morphological and metabolic changes, massive nuclear fragmentation, chromatin condensation and the activation of specific proteins (30). Therefore, the stimulation of apoptosis remains important in cancer eradication. Data generated in the present study indicated that the numbers of apoptotic bodies and TUNEL-positive cells increased following shikonin treatment, representing the cytotoxic effect of this compound that is mediated through the apoptotic pathway (Fig. 2).

Mitochondria and cytochrome c, a key electron carrier in the electron transport chain, have a crucial function in apoptosis induced by various stimuli (26). During the apoptotic process the $\Delta \psi \mathrm{m}$ is destroyed, leading to the leakage of cytochrome $\mathrm{c}$ from mitochondria into the cytosol. This process is regulated by the pro-survival/anti-apoptotic protein $\mathrm{Bcl}-2$ and pro-apoptotic protein Bax, and results in the activation and heptamerization of the adaptor molecule apoptotic protease-activating factor 1 (Apaf-1), forming the apoptosome complex. Apaf-1 then cleaves the preform of caspase- 9 to initiate the caspase cascade, resulting in apoptosis (31). Therefore, mitochondria-mediated apoptosis involves upregulation of Bax, downregulation of Bcl-2, disruption of mitochondrial membrane permeability, leakage of cytochrome $c$ into the cytosol, the formation of apoptotic bodies, and initiation of the caspase cascade. Upon shikonin treatment, mitochondrial membrane depolarization was observed. The level of Bcl-2 was decreased and that of Bax was increased. These changes in protein expression altered the mitochondria membrane permeability and caused release of cytochrome $c$ into the cytosol, inducing apoptosis via caspase-9 and -3 cleavage (Fig. 2). This indicates that shikonin initiates mitochondria-mediated apoptosis.

Multiple studies have demonstrated that the cytotoxic proprieties of the majority of anticancer drugs are associated with the regulation of MAPK-family protein activity (32-34). The present study also investigated the involvement of MAPKs in shikonin-induced apoptosis. Levels of phosphorylated JNK, p38, and ERK were increased following shikonin treatment in a time-dependent manner, and specific inhibitors of these kinases attenuated the decrease in A2780-CR cell viability caused by shikonin (Fig. 3). These results suggested that MAPK-mediated pathways are activated in shikonin-induced apoptosis of A2780-CR cells.

EMT is associated with tumor metastasis $(35,36)$. EMT promotes cancer cell migration and invasion and has a crucial function in the invasion and metastasis of epithelial cancer specifically $(35,36)$. EMT is associated with the downregulation of epithelial markers (including E-cadherin and $\beta$-catenin) and upregulation of interstitial markers (including $\mathrm{N}$-cadherin and fibronectin). Alterations in N-cadherin levels, as well as the associated EMT E-cadherin/N-cadherin switching, are considered to be risk markers for tumor progression and invasion (37). These results have been previously assessed 
for gastric, prostate and oral carcinoma cell lines $(38,39)$. E-cadherin/N-cadherin switching is essential for increased cell motility, but not for the morphological alterations that occur in the EMT process (37). There are limited reports regarding EMT in ovarian cancer. Ovarian cancer cells easily invade and diffuse in the pelvic cavity, and this results in the high mortality rate of ovarian cancer $(2,3,36)$. EMT is an early event in the invasion and metastasis of cancer cells, including ovarian cancer cells $(36,40)$. The present study revealed that the migration of A2780-CR cells was significantly decreased upon shikonin treatment, indicating that EMT decreases cell polarity and normal cell-cell adhesion, induces the loss of epithelial characteristics and improves the invasion of the cells. The level of E-cadherin (an epithelial marker) was enhanced, while the level of $\mathrm{N}$-cadherin (an interstitial marker) was decreased following shikonin treatment, indicating that shikonin attenuates EMT.

Taken together, these results suggested that A2780-CR ovarian cancer cells are highly sensitive to shikonin, which activates apoptosis and attenuates EMT. Therefore, shikonin should be considered a promising chemotherapeutic candidate to overcome cisplatin resistance in ovarian cancer.

\section{Acknowledgements}

The present study was supported by grants from National Research Foundation of Korea funded by the Korean Government (grant nos. NRF-2016R1A2B4007934 and NRF-2015M2A2A7061657).

\section{References}

1. Liu X, Gao Y, Lu Y, Zhang J, Li L and Yin F: Oncogenes associated with drug resistance in ovarian cancer. J Cancer Res Clin Oncol 141: 381-395, 2015.

2. Suh DH, Kim M, Kim HJ, Lee KH and Kim JW: Major clinical research advances in gynecologic cancer in 2015. J Gynecol Oncol 27: e53, 2016.

3. American Cancer Society. Chemotherapy for ovarian cancer, http://www.cancer.org/cancer/ovariancancer/detailed guide/ovarian-cancer-treating-chemotherapy 2014. Accessed February 4, 2016).

4. Huang H, Tong TT, Yau LF, Chen CY, Mi JN, Wang JR and Jiang ZH: LC-MS based sphingolipidomic study on A2780 human ovarian cancer cell line and its taxol-resistant strain. Sci Rep 6: 34684, 2016

5. Galluzzi L, Senovilla L, Vitale I, Michels J, Martins I, Kepp O, Castedo M and Kroemer G: Molecular mechanisms of cisplatin resistance. Oncogene 31: 1869-1883, 2012.

6. Hiss D: Optimizing molecular-targeted therapies in ovarian cancer: The renewed surge of interest in ovarian cancer biomarkers and cell signaling pathways. J Oncol 2012: 737981, 2012.

7. Kim H, Park GS, Lee JE and Kim JH: A leukotriene B4 receptor-2 is associated with paclitaxel resistance in MCF-7/DOX breast cancer cells. Br J Cancer 109: 351-359, 2013.

8. Kim DK, Seo EJ, Choi EJ, Lee SI, Kwon YW, Jang IH, Kim SC, Kim KH, Suh DS, Seong-Jang K, et al: Crucial role of HMGA1 in the self-renewal and drug resistance of ovarian cancer stem cells. Exp Mol Med 48: e255, 2016.

9. Huang WR, Zhang Y and Tang X. Shikonin inhibits the proliferation of human lens epithelial cells by inducing apoptosis through ROS and caspase-dependent pathway. Molecules 19: 7785-7797, 2014.

10. Liu T, Ma C, Yang L, Wang W, Sui X, Zhao C and Zu Y: Optimization of shikonin homogenate extraction from Arnebia euchroma using response surface methodology. Molecules 18: 466-481, 2013

11. Andújar I, Ríos JL, Giner RM and Recio MC: Pharmacological properties of shikonin - A review of literature since 2002. Planta Med 79: 1685-1697, 2013
12. Han CT, Kim MJ, Moon SH, Jeon YR, Hwang JS, Nam C, Park CW, Lee SH, Na JB, Park CS, et al: Acute and 28-day subacute toxicity studies of hexane extracts of the roots of Lithospermum erythrorhizon in Sprague-Dawley rats. Toxicol Res 31: 403-414, 2015

13. He G, He G, Zhou R,Pi Z, Zhu T, Jiang L and Xie Y: Enhancement of cisplatin-induced colon cancer cells apoptosis by shikonin, a natural inducer of ROS in vitro and in vivo. Biochem Biophys Res Commun 469: 1075-1082, 2016.

14. Jeung YJ, Kim HG, Ahn J, Lee HJ, Lee SB, Won M, Jung CR, Im JY, Kim BK, Park SK, et al: Shikonin induces apoptosis of lung cancer cells via activation of FOXO3a/EGR1/SIRT1 signaling antagonized by p300. Biochim Biophys Acta 1863: 2584-2593, 2016.

15. Jing H, Sun W, Fan J, Zhang Y, Yang J, Jia J, Li J, Guo J, Luo S and Zheng Y: Shikonin induces apoptosis of HaCaT cells via the mitochondrial, Erk and Akt pathways. Mol Med Rep 13: 3009-3016, 2016.

16. Ko H, Kim SJ, Shim SH, Chang $\mathrm{H}$ and $\mathrm{Ha} \mathrm{CH}$ : Shikonin induces apoptotic cell death via regulation of p53 and Nrf2 in AGS human stomach carcinoma cells. Biomol Ther (Seoul) 24: 501-509, 2016.

17. Lu D, Qian J, Li W, Feng Q, Pan S and Zhang S: $\beta$-hydroxyisovaleryl-shikonin induces human cervical cancer cell apoptosis via PI3K/AKT/mTOR signaling. Oncol Lett 10: 3434-3442, 2015.

18. Tang X, Zhang C, Wei J, Fang Y, Zhao R and Yu J: Apoptosis is induced by shikonin through the mitochondrial signaling pathway. Mol Med Rep 13: 3668-3674, 2016.

19. Trivedi R, Müller GA, Rathore MS, Mishra DP and Dihazi H: Anti-leukemic activity of shikonin: Role of ERP57 in shikonin induced apoptosis in acute myeloid leukemia. Cell Physiol Biochem 39: 604-616, 2016.

20. Wei Y, Li M, Cui S, Wang D, Zhang CY, Zen K and Li L: Shikonin inhibits the proliferation of human breast cancer cells by reducing tumor-derived exosomes. Molecules 21: pii: E777, 2016.

21. Christiansen JJ and Rajasekaran AK: Reassessing epithelial to mesenchymal transition as a prerequisite for carcinoma invasion and metastasis. Cancer Res 66: 8319-8326, 2006.

22. Yin SY, Peng AP, Huang LT, Wang YT, Lan CW and Yang NS: The phytochemical shikonin stimulates epithelial-mesenchymal transition (EMT) in skin wound healing. Evid Based Complement Alternat Med 2013: 262796, 2013.

23. Thakur R, Trivedi R, Rastogi N, Singh M and Mishra DP: Inhibition of STAT3, FAK and Src mediated signaling reduces cancer stem cell load, tumorigenic potential and metastasis in breast cancer. Sci Rep 5: 10194, 2015.

24. Zhang FY, Hu Y, Que ZY, Wang P, Liu YH, Wang ZH and Xue YX: Shikonin inhibits the migration and invasion of human glioblastoma cells by targeting phosphorylated $\beta$-catenin and phosphorylated PI3K/Akt: A potential mechanism for the anti-glioma efficacy of a traditional Chinese herbal medicine. Int J Mol Sci 16: 23823-23848, 2015.

25. Carmichael J, DeGraff WG, Gazdar AF, Minna JD and Mitchell JB: Evaluation of a tetrazolium-based semiautomated colorimetric assay: Assessment of chemosensitivity testing. Cancer Res 47: 936-942, 1987.

26. Garrido C, Galluzzi L, Brunet M, Puig PE, Didelot C and Kroemer G: Mechanisms of cytochrome c release from mitochondria. Cell Death Differ 13: 1423-1433, 2006.

27. Bi W, Wang Y, Sun G, Zhang X, Wei Y, Li L and Wang X: Paclitaxel-resistant HeLa cells have up-regulated levels of reactive oxygen species and increased expression of taxol resistance gene 1. Pak J Pharm Sci 27: 871-878, 2014.

28. Okon IS and Zou MH: Mitochondrial ROS and cancer drug resistance: Implications for therapy. Pharmacol Res 100: 170-174, 2015.

29. Wangpaichitr M, Wu C, Li YY, Nguyen DJM, Kandemir H, Shah S, Chen S, Feun LG, Prince JS, Kuo MT and Savaraj N: Exploiting ROS and metabolic differences to kill cisplatin resistant lung cancer. Oncotarget 8: 49275-49292, 2017.

30. Peitsch MC, Polzar B, Stephan H, Crompton T, MacDonald HR, Mannherz HG and Tschopp J: Characterization of the endogenous deoxyribonuclease involved in nuclear DNA degradation during apoptosis (programmed cell death). EMBO J 12: 371-377, 1993.

31. Desagher $S$ and Martinou JC: Mitochondria as the central control point of apoptosis. Trends Cell Biol 10: 369-377, 2000. 
32. Sun Y, Liu WZ, Liu T, Feng X, Yang N and Zhou HF: Signaling pathway of MAPK/ERK in cell proliferation, differentiation, migration, senescence and apoptosis. J Recept Signal Transduct Res 35: 600-604, 2015.

33. Habli Z, Toumieh G, Fatfat M, Rahal ON and Gali-Muhtasib H: Emerging cytotoxic alkaloids in the battle against cancer: Overview of molecular mechanisms. Molecules 22: pii: E250, 2017.

34. Xing K and Lisong S: Molecular targeted therapy of cancer: The progress and future prospect. Front Lab Med 1: 69-75, 2017.

35. Chen ZS, Ling DJ, Zhang YD, Feng JX, Zhang XY and Shi TS: Octamer-binding protein 4 affects the cell biology and phenotypic transition of lung cancer cells involving $\beta$-catenin/ E-cadherin complex degradation. Mol Med Rep 11: 1851-1858, 2015.

36. Stewart CJ and McCluggage WG: Epithelial-mesenchymal transition in carcinomas of the female genital tract. Histopathology 62 : 31-43, 2013.

37. Maeda M, Johnson KR and Wheelock MJ: Cadherin switching: Essential for behavioral but not morphological changes during an epithelium-to-mesenchyme transition. J Cell Sci 118: 873-887, 2005.
38. DI Domenico M, Pierantoni GM, Feola A, Esposito F, Laino L, DE Rosa A, Rullo R, Mazzotta M, Martano M, Sanguedolce F, et al: Prognostic significance of N-Cadherin expression in oral squamous cell carcinoma. Anticancer Res 31: 4211-4218, 2011.

39. Liu LK, Jiang XY, Zhou XX, Wang DM, Song XL and Jiang HB: Up-regulation of vimentin and aberrant expression of E-cadherin/beta-catenin complex in oral squamous cell carcinomas: Correlation with the clinicopathological features and patient outcome. Mod Pathol 23: 213-224, 2010.

40. Powell CD, Paullin TR, Aoisa C, Menzie CJ, Ubaldini A and Westerheide SD: The heat shock transcription factor HSF1 induces ovarian cancer epithelial-mesenchymal transition in a 3D spheroid growth model. PLoS One 11: e0168389, 2016.

\footnotetext{
(7) $\odot$ This work is licensed under a Creative Common cc) Attribution-NonCommercial-NoDerivatives 4.0 International (CC BY-NC-ND 4.0) License.
} 